\title{
Linkage between toxin production and purine biosynthesis in Clostridium difficile
}

\author{
TSUNEO MAEGAWA, TADAHIRO KARASAWA, TOSHIKO OHTA*, XINGMIN WANG, HARU KATO, \\ HIDEO HAYASHI ${ }^{\dagger}$ and SHINICHI NAKAMURA
}

Department of Bacteriology, School of Medicine, Kanazawa University, Kanazawa 920-8640, * College of Medical Technology and Nursing and †Institute of Basic Medical Sciences, University of Tsukuba, Tsukuba 305, Japan

\begin{abstract}
The production of toxins $\mathbf{A}$ and $\mathbf{B}$ by Clostridium difficile was greatly enhanced under biotin-limited conditions, in which a 140-kDa protein was expressed strongly. Gene cloning revealed that this protein was a homologue of formylglycinamidine ribonucleotide synthetase (FGAM synthetase, EC 6.3.5.3), which is known as PurL in Escherichia coli and catalyses the fourth step of the de novo purine biosynthesis pathway. This enzyme consisted of a single polypeptide, although FGAM synthetases of gram-positive bacteria usually consist of two subunits. Inhibition of the enzymic activity of $C$. difficile PurL by $O$-diazoacetyl-L-serine (azaserine) resulted in enhanced toxin B production even in biotin-sufficient conditions. In contrast, blockade of the preceding step of the PurL catalysing step by sulfamethoxazole inhibited toxin B production almost completely. These results suggest that accumulation of formylglycinamide ribonucleotide (FGAR), a substrate of FGAM synthetase, enhances toxin production by $C$. difficile and depletion of FGAR reduces toxin production.
\end{abstract}

\section{Introduction}

Clostridium difficile produces two toxins, A (enterotoxin) and B (cytotoxin), which are causally involved in $C$. difficile-associated diarrhoea (CDAD) and pseudomembranous colitis (PMC) [1-3]. The genes of both toxins have been cloned and sequenced [4-6]. The enzymic activity of both toxins is glycosyltransferase, which modifies small GTP-binding proteins of the target cells [7-9].

The production of toxins by $C$. difficile is affected by its growth conditions, especially by various environmental stresses. Toxin levels in culture supernates are increased by raising the temperature or the oxidationreduction potential, or by adding subinhibitory concentrations of antibiotics [10-12]. Previous studies showed that the production of both toxins by $C$. difficile is affected by the nutritional conditions in defined media [13-16]. In particular, critical limitation of biotin concentration strongly enhanced production of both toxins while bacterial growth was reduced (biotin

Received 19 March 2001; revised version accepted 21 June 2001.

Corresponding author: Professor S. Nakamura (e-mail: nakamura@med.kanazawa-u.ac.jp). effect) [13]. The biotin effect is the most potent toxinenhancing stress known to date. Recently, another research group confirmed that biotin limitation elevated the ratio of toxin production to total protein synthesis [17]. The biotin effect was neutralised by adding excess biotin, glutamine, glutamic acid, asparagine or lysine [14], suggesting that the insufficiency of biotin influences toxin production through bacterial metabolism, especially amino acid metabolism.

As the biotin effect is a general response observed in all toxigenic $C$. difficile strains [13], it is a good model for studying the regulatory mechanisms of toxin production by $C$. difficile. In this study, a $140-\mathrm{kDa}$ protein expressed strongly under biotin-limited condition was investigated for its role in de novo purine biosynthesis and the effects of some inhibitors of the enzymes involved in purine biosynthesis on toxin B production were examined. A hypothesis for a key mechanism of the biotin effect is discussed.

\section{Materials and methods}

\section{Chemicals}

$O$-Diazoacetyl-L-serine (azaserine) and sulfamethoxazole were obtained from Sigma. All chemicals and 
reagents were from commercial sources and were molecular biology grade or the highest available grade.

\section{C. difficile strains and defined media}

C. difficile strain KZ 1647, which has shown a typical response of enhanced production of toxins under biotin-limited condition $[13,14]$, was used for most experiments. Sixty-one toxigenic and 41 non-toxigenic strains of $C$. difficile that had been kept in this laboratory were also used $[18,19]$.

A defined medium used in this study was prepared as described by Yamakawa et al. [13] except that the concentrations of minerals were modified according to Karasawa et al. [20]. The concentrations of biotin were $50 \mathrm{nM}(12.2 \mu \mathrm{g} / \mathrm{L})$ in biotin-sufficient conditions and $50 \mathrm{pM}$ in biotin-limited conditions $[13,14]$. Inoculation of $C$. difficile strains and all other anaerobic manipulations were performed as described previously [13]. Cultures were incubated at $37^{\circ} \mathrm{C}$, and bacterial growth was monitored by measuring the optical density (OD) at $560 \mathrm{~nm}$.

\section{Toxin B assay}

The filtrates of 5-7-day-old cultures were used for cytotoxicity (mainly caused by toxin B) assays by the conventional 96-well microtitration plate method with a mouse fibroblast cell line (L929). The highest dilution that caused $100 \%$ cell rounding after incubation for $24 \mathrm{~h}$ was used to define the number of cytotoxic units (CU) [13].

\section{Intracellular proteins and protein sequencing}

KZ 1647 cells were washed twice with $\mathrm{NaCl} 0.85 \%$ and homogenised by vortex mixing with glass beads $(0.1 \mathrm{~mm}$ diameter, $1.5 \mathrm{~g} / \mathrm{ml})$ in a buffer containing $5 \mathrm{~mm}$ Tris-HCl (pH 7.5), $5 \mathrm{~mm}$ EDTA, $1 \mathrm{~mm}$ phenylmethylsulfonyl fluoride. The homogenates were centrifuged at $240000 \mathrm{~g}$ for $30 \mathrm{~min}$ at $4^{\circ} \mathrm{C}$ to remove cell debris and membrane components. Protein concentration was estimated by the method of Bradford [21] with bovine serum albumin as a standard.

The intracellular proteins were subjected to standard SDS-PAGE with acrylamide $5 \%$ or $10 \%$ gels. Gels were stained with Coomassie Brilliant Blue R-250. A $140-\mathrm{kDa}$ band to be analysed was cut from several 5\% gels. The protein was eluted in $10 \mathrm{~mm}$ phosphatebuffered saline (PBS, pH 7.0) containing SDS 0.1\%, 2mercaptoethanol (ME) 2\%, $5 \mathrm{mM}$ EDTA and then dialysed against $10 \mathrm{mM}$ phosphate buffer $(\mathrm{pH} 7.0)$ containing $2 \mathrm{ME} 1 \%, 5 \mathrm{mM}$ EDTA. The extracted protein was N-terminally sequenced with an Applied Biosystems Model 476A Sequencer (Applied Biosystems, Foster City, CA, USA).

\section{DNA techniques}

Standard recombinant DNA techniques were used [22]. Oligonucleotides were synthesised by Hokkaido System Science (Sapporo, Japan). An Escherichia coli K12 derivative strain, TOP10F' (Invitrogen, Carlsbad, CA, USA), was grown in Luria-Bertani broth or on LuriaBertani agar. Ampicillin $100 \mathrm{mg} / \mathrm{L}$ was added as necessary.

PCR was performed with a GeneAmp PCR System 9700 Thermal Cycler (Applied Biosystems) and AmpliTaq (Applied Biosystems) or Ex Taq (Takara, Tokyo, Japan) DNA polymerase. The TA cloning system (pCR 2.1 vector; Invitrogen) was used for subcloning PCR products. DNA sequencing was performed with the BigDye Terminator Cycle Sequencing Kit (Applied Biosystems) and an ABI Prism 310 Genetic Analyser (Applied Biosystems).

For preparation of $C$. difficile DNA, bacterial cells grown in GAM broth (Nissui, Tokyo, Japan) were treated with mutanolysin and proteinase $\mathrm{K}$ (Nacalai tesque, Kyoto, Japan) and lysed by adding SDS to a final concentration of $1 \%$. The lysate was then extracted with phenol and precipitated with ethanol.

For Southern blot analysis, the genomic DNA from strain KZ 1647 was digested completely with EcoRI, HindIII, Pst I, Sau3AI or Xba I (Takara), separately, resolved in an agarose $0.8 \%$ gel and then transferred to a positively charged nylon membrane (Hybond-N+; Amersham Pharmacia Biotech, Uppsala, Sweden) under alkaline conditions. DNA probes were directly labelled with a thermostable alkaline phosphatase enzyme by the AlkPhos Direct System (Amersham Pharmacia Biotech). The hybridised probe was visualised with Enhanced Chemifluorescence (ECF) Substrate (Amersham Pharmacia Biotech) and a FluorImager 595 Imaging System (Amersham Pharmacia Biotech). Labelling, hybridisation and detection were performed according to the manufacturer's instructions.

To amplify unknown DNA sequences contiguous to known loci, the single specific primer-PCR (SSPPCR) technique $[23,24]$ was used. The genomic DNA from strain KZ 1647 was digested with HindIII, Sau3AI or XbaI, separately, and ligated to pKF3 plasmid DNA (Takara) digested with the same restriction endonucleases. Small samples of each ligation mixture were used as templates for PCR amplification with gene-specific primers and pKF3-specific primers, and nested PCR was performed as necessary. The final PCR products were sequenced from both ends to confirm whether the desired contiguous sequences were actually amplified. PCR primers used in each SSP-PCR step were designed based on the newly obtained sequence information. 


\section{Polyclonal antibody against C. difficile PurL}

The DNA region corresponding to the amino acid residues 1-407 of $C$. difficile PurL (see Results) was amplified by PCR. This PCR product was subcloned into pGEX-2T (Amersham Pharmacia Biotech) expression vector and verified by sequencing. The glutathione S-transferase (GST) fusion protein from the E. coli lysate was bound to Glutathione-Sepharose 4B Matrix (Amersham Pharmacia Biotech) and washed with PBS containing Tween $200.1 \%$. Without further elution or thrombin-cleavage steps, the protein-bound matrix was directly injected intraperitoneally (i.p.) and subcutaneously (s.c.) into BALB/c mice (5 $\mu \mathrm{g}$ of protein/ mouse). The mice were given a booster injection (i.p.) of the same protein-bound matrix on days 14 and 42 after the first injection $(2.5 \mu \mathrm{g}$ of protein/mouse). A week after the final injection, each mouse was given an i.p. injection of $0.5 \mathrm{ml}$ of 2,6,10,14-tetramethylpentadecane (pristane; Sigma); 10 days later, ascites were induced by injecting (i.p.) SP2/0-Ag14 mouse myeloma cells $\left(2.0 \times 10^{8}\right.$ cells/mouse $)$. The harvested, pooled ascites fluids were absorbed with the GlutathioneSepharose 4B matrix bound with a GST-tag only, and used as a polyclonal antibody against $C$. difficile PurL.

For Western blot analysis, proteins separated on an SDS-PAGE gel were transferred to a polyvinylidene difluoride (PVDF) membrane (PVDF-Plus; MSI, Westboro, MA, USA) with a semi-dry apparatus (Atto, Tokyo, Japan). An alkaline phosphatase-conjugated goat anti-mouse IgG antibody (Sigma) was used as a secondary antibody. Immunochemical signals were detected with ECF substrate and a FluorImager 595 imaging system (Amersham Pharmacia Biotech).

\section{Results}

\section{Differential expression of proteins under biotin- limited conditions}

The intracellular proteins from $C$. difficile KZ 1647 cells grown in biotin-sufficient ( $50 \mathrm{nM}$ biotin) and biotin-limited (50 pM biotin) conditions were compared by standard SDS-PAGE and two-dimensional PAGE. Several large bands $(>120 \mathrm{kDa})$ were observed only in the biotin-limited conditions (Fig. 1). Among these large bands, a $140-\mathrm{kDa}$ band was observed most clearly and was not recognised by either antitoxin A or antitoxin B serum in Western blotting experiments (data not shown). This indicated that the $140-\mathrm{kDa}$ protein was neither one of the toxins nor the degraded polypeptides of the toxins. It was likely to be expressed as a result of a physiological response to biotin limitation. Although this protein made a stringy and diffused spot on a two-dimensional gel, it was separated efficiently by conventional SDS-PAGE when the concentration of acrylamide was reduced to $5 \%$ (data not shown). N-Terminal sequencing of this protein demonstrated the sequence MLNTENKDS

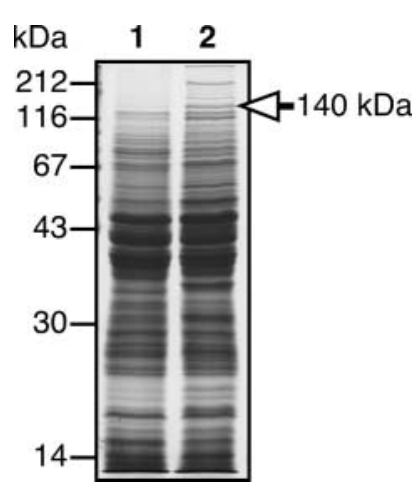

Fig. 1. SDS-PAGE (10\%) of each intracellular protein preparation $(100 \mu \mathrm{g})$ from $C$. difficile $\mathrm{KZ} 1647$ cells grown in (1) biotin-sufficient (50 nM biotin) or (2) biotin-limited ( $50 \mathrm{pM}$ biotin) conditions for $30 \mathrm{~h}$. The $140-\mathrm{kDa}$ protein band is indicated by the arrow.

MVRRVLVEKR; no sequences homologous to this amino acid sequence were found in databases.

\section{Cloning of the 140-kDa protein gene}

The DNA region corresponding to the N-terminal amino acid sequence was cloned by PCR. Two degenerate primers of KAG46D, 5'-ATGYTNAA YACNGARAAYAA-3' (corresponding to the amino acid sequence MLNTENK) and KAG47DI, 5'-CKY TTYTCIACIARNACNC-3' (complementary nucleotide sequence corresponding to the amino acid sequence RVLVEKR) were used for amplification. Both primers had 256-fold degeneracy. A PCR fragment of c. $60 \mathrm{bp}$ was cloned into pCR2.1 and sequenced to obtain the inside sequence information between the primers. With the nucleotide sequence information corresponding to the amino acid sequence DSMVR as a first clue, genome walking by SSP-PCR was performed. A $3807-$ bp DNA sequence of an open reading frame (ORF) was obtained from three SSP-PCR fragments. This ORF encoded a polypeptide of 1268 amino acids with a calculated mol. wt of 140645 , which agreed with the molecular size determined by SDS-PAGE. The Nterminal amino acid sequence deduced from the DNA sequence was exactly the same as that from the Edman degradation analysis. Furthermore, putative ShineDalgarno and rho-independent terminator sequences were found upstream and downstream of the ORF, respectively.

The amino acid sequence of the $140-\mathrm{kDa}$ protein deduced from the DNA sequence showed apparent homologies to several formylglycinamidine ribonucleotide synthetases (FGAM synthetase, EC 6.3.5.3), which catalyse the fourth step of the de novo purine biosynthesis pathway. In addition to the overall homologies, there was a conserved motif of the glutamine amidotransferases active site (PROSITE database accession no. PS00442) [25] (Fig. 2) that is consistent with the function of FGAM synthetase. Furthermore, two other characteristic motifs that are highly conserved in many FGAM synthetases were also 
found. One was BL00442B (Blocks database) [26] and the other was PD009676 (ProDom database) [27]. BL00442A (Blocks database) was also found, but it was obviously equivalent to the PS00442 (Fig. 2). Therefore, it was concluded that this ORF encodes a homologue of FGAM synthetase. The location of those conserved motifs and the overall homologies indicated that the subunit structure of the $C$. difficile FGAM synthetase was more similar to that of E. coli (PurL) than to Bacillus subtilis (PurL and PurQ) (Fig. 2), so this gene was designated as purL.

The sequence of the $C$. difficile purL reported in this paper has been deposited in the DDBJ/EMBL/GenBank databases under accession no. AB047389.

\section{Distribution of purL among C. difficile strains}

A pair of primers was designed to amplify the coding region (from initiation ATG to termination TAA) of purL. All 62 toxigenic and 41 non-toxigenic strains of C. difficile (including KZ 1647) generated single PCR fragments of the same size regardless of toxigenicity or source of strains (Fig. 3a). Representative strains were further examined by restriction endonuclease digestion of the PCR products and were confirmed to possess

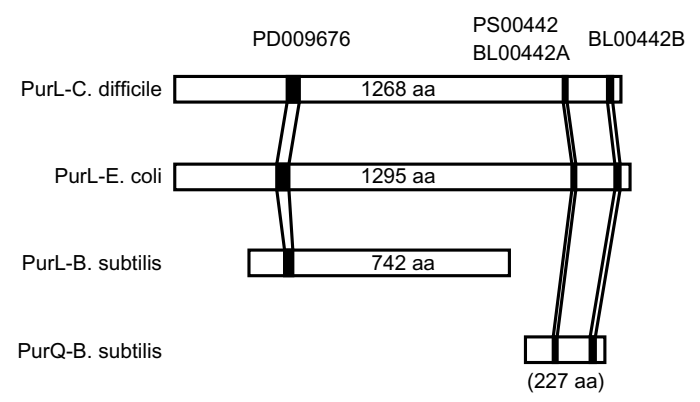

Fig. 2. Schematic representation of FGAM synthetase polypeptides. The characteristic motifs for FGAM synthetases are indicated by black regions. The amino acid sequence data of $E$. coli and B. subtilis were obtained from the SWISS-PROT database.

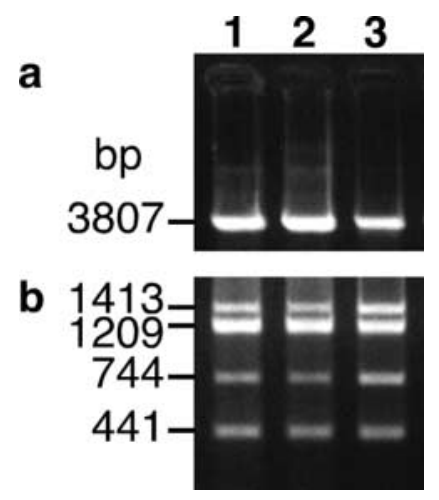

Fig. 3. PCR detection of the purL gene from $C$. difficile strains. The entire coding region (from initiation ATG to termination TAA) of purL was amplified (a) and PCR products were digested with NdeI (b). Lane 1, KZ 1647 (toxigenic strain); 2, VPI 10463 (toxigenic strain); 3, ATCC 43597 (nontoxigenic strain).
purL (Fig. 3b). Southern blot analysis indicated that purL was present in one copy per genome of strain $\mathrm{KZ}$ 1647 (data not shown).

\section{Expression of PurL under biotin-sufficient and biotin-limited conditions}

To study the expression of FGAM synthetase protein (PurL) in $C$. difficile, a polyclonal antibody was prepared for Western blot analyses. A recombinant polypeptide of amino acid residues 1-407 of PurL was used as an antigen. The antibody raised reacted with the $140-\mathrm{kDa}$ band specifically, as expected (Fig. 4).

In biotin-sufficient conditions (50 nM biotin), PurL was found after incubation for $18 \mathrm{~h}$ (at the mid-exponential growth phase) and was barely detected after incubation for $\geqslant 24 \mathrm{~h}$. However, in biotin-limited conditions (50 pM biotin), PurL was observed between 18-h and 48 - $h$ incubation periods (from the early exponential phase to the decline phase), reaching a maximum at $30 \mathrm{~h}$ (the late exponential phase) (Fig. 4). These results show that the differences in PurL expression between the biotin-sufficient and biotin-limited conditions are most evident in the late stationary to decline phase of the growth cycle.

\section{Involvement of PurL and purine biosynthesis in the production of toxin $B$}

The fact that the expression pattern of PurL differed between the biotin-sufficient and biotin-limited conditions (Fig. 4) indicates that some alteration of purine biosynthesis might enhance toxin production. Therefore, a range of chemicals was added to the defined medium to clarify the relationships among toxin production, PurL and purine biosynthesis.

Purine bases and purine nucleosides. Adenine, hypoxanthine, adenosine or guanosine (1 mM) was added individually to a defined medium. Guanine was omitted because of its very low solubility in the medium. None of the purine compounds showed any significant effect

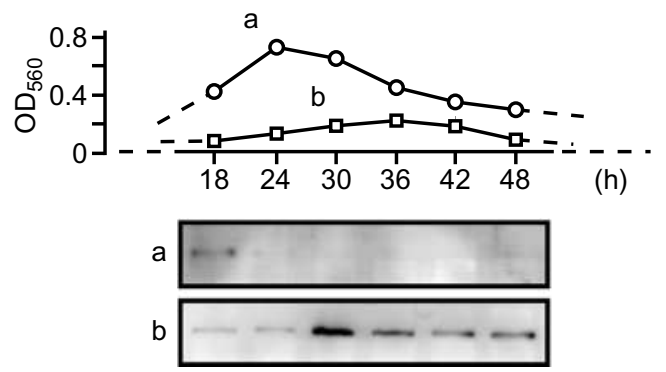

Fig. 4. Time course of PurL expression. Each intracellular protein preparation $(20 \mu \mathrm{g})$ from $C$. difficile $\mathrm{KZ} 1647$ cells grown in (a) biotin-sufficient (50 nM biotin) or (b) biotinlimited (50 pM biotin) conditions for the indicated time was examined by Western blotting with an anti-PurL antibody. The growth curves are represented in the upper part. Western blot experiments were not done before $18 \mathrm{~h}$ and after $48 \mathrm{~h}$. 
on the production of toxin $\mathrm{B}$ in either biotin-sufficient or biotin-limited conditions.

Glutamine antagonist. Glutamine antagonists are potent inhibitors of several glutamine-dependent amidotransferases; $O$-diazoacetyl-L-serine (azaserine), one of the authentic glutamine antagonists, was reported to inhibit FGAM synthetase almost specifically [28]. In biotin-sufficient condition, the addition of sublethal doses of azaserine resulted in decreased growth and moderately increased toxin B production (Table 1). When one of the purine compounds $(1 \mathrm{mM})$ was added simultaneously to the medium, toxin B production was enhanced more strongly (Table 1). In particular, when hypoxanthine or adenosine was added to a medium containing $15 \mu \mathrm{M}$ azaserine, toxin $\mathrm{B}$ production was increased to the same level as that of the biotin effect (Table 1). In biotin-limited conditions, the addition of azaserine decreased both growth and toxin B production dose-dependently $(1-25 \mu \mathrm{M})$. When $1 \mathrm{mM}$ adenosine was added to the medium containing $5 \mu \mathrm{M}$ azaserine, toxin $\mathrm{B}$ production recovered to the same level as that without azaserine (Table 1).

Sulfonamide. Sulfonamides inhibit folic acid biosynthesis and result in the inhibition of purine biosynthesis. Bacterial growth and toxin production were completely inhibited by adding $>500 \mu \mathrm{M}(126.65 \mathrm{mg} / \mathrm{L})$ of sulfamethoxazole to the defined medium (Table 1). This inhibitory effect on growth was partly neutralised by adding $1 \mathrm{~mm}$ adenosine. When $1 \mathrm{~mm}$ adenosine and $500 \mu \mathrm{M}$ sulfamethoxazole were added to the medium, the bacterial growth was almost half the level of that without sulfamethoxazole. However, the production of toxin B was still almost completely inhibited (Table 1). This inhibitory effect on toxin B production was observed constantly; it was not affected by changing biotin concentration or adding azaserine, or both (Table 1).
The degrees of PurL expression under the growth conditions described above were examined in the late stationary to decline growth phase and were in good agreement with the potency of toxin production. PurL was expressed strongly in the toxin-enhancing conditions $(50 \mathrm{pM}$ biotin and $50 \mathrm{nM}$ biotin $+15 \mu \mathrm{M}$ azaserine) (Fig. 5b, lanes 2 and 3) and was expressed weakly in the toxin-reducing conditions $(50 \mathrm{pM}$ biotin $+500 \mu \mathrm{M}$ sulfamethoxazole $+1 \mathrm{mM}$ adenosine and $50 \mathrm{pM}$ biotin $+10 \mathrm{mM}$ glutamine) (Fig. 5b, lanes 4 and 5). However, in the exponential growth phase, PurL expression was similar under the various conditions tested (Fig. 5a).

\section{Discussion}

The FGAM synthetase gene, purL, of which the product was expressed strongly under biotin-limited conditions in $C$. difficile, was cloned. The de novo purine biosynthesis pathway includes 10 enzymic steps leading from phosphoribosylpyrophosphate (PRPP) to inosine monophosphate (IMP). FGAM synthetase is located on the fourth step of this pathway (Fig. 6) and

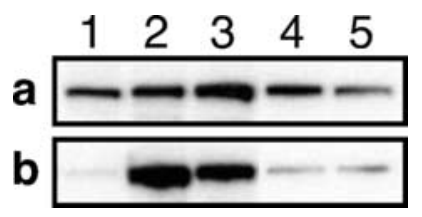

Fig. 5. PurL expression under various culture conditions. Each intracellular protein preparation $(20 \mu \mathrm{g})$ from $C$. difficile $\mathrm{KZ}$ 1647 cells grown under various toxin-enhancing or toxinreducing conditions was examined by Western blotting with an anti-PurL antibody. Panel a, exponential growth phase; b, late stationary to decline growth phase. Lane $1,50 \mathrm{nM}$ biotin (biotin-sufficient condition, control); 2, $50 \mathrm{pM}$ biotin (biotinlimited condition); 3, $50 \mathrm{nM}$ biotin $+15 \mu \mathrm{M}$ azaserine; 4, $50 \mathrm{pM}$ biotin $+500 \mu \mathrm{M}$ sulfamethoxazole $+1 \mathrm{mM}$ adenosine; $\mathbf{5}, 50 \mathrm{pm}$ biotin $+10 \mathrm{mM}$ glutamine.

Table 1. Toxin B production by $C$. difficile $\mathrm{KZ} 1647$

\begin{tabular}{|c|c|c|}
\hline \multirow[b]{2}{*}{ Chemicals added } & \multicolumn{2}{|c|}{ Toxin B titre* (growth $^{\dagger}$ ) with } \\
\hline & $50 \mathrm{nM}$ biotin & $50 \mathrm{pM}$ biotin \\
\hline None (basal composition) & $4(+++)$ & $4096(+)$ \\
\hline $10 \mathrm{mM}$ glutamine & $4(+++)$ & $2(++)$ \\
\hline $1 \mu \mathrm{M}$ azaserine & $8(+++)$ & $4096(+)$ \\
\hline $5 \mu \mathrm{M}$ azaserine & $64(++)$ & $256(+)$ \\
\hline $15 \mu \mathrm{M}$ azaserine & $128(++)$ & $-(-)$ \\
\hline $25 \mu \mathrm{M}$ azaserine & $-(-)$ & $-(-)$ \\
\hline $5 \mu \mathrm{M}$ azaserine $+1 \mathrm{mM}$ adenosine & ND & $8192(+)$ \\
\hline $15 \mu \mathrm{M}$ azaserine $+1 \mathrm{mM}$ adenine & $256(++)$ & ND \\
\hline $15 \mu \mathrm{M}$ azaserine $+1 \mathrm{mM}$ hypoxanthine & $4096(++)$ & ND \\
\hline $15 \mu \mathrm{M}$ azaserine $+1 \mathrm{mM}$ adenosine & $4096(++)$ & ND \\
\hline $15 \mu \mathrm{M}$ azaserine $+1 \mathrm{mM}$ guanosine & $256(++)$ & ND \\
\hline $250 \mu \mathrm{M}$ sulfamethoxazole & $4(++)$ & $4(+)$ \\
\hline $500 \mu \mathrm{M}$ sulfamethoxazole & $-(-)$ & $-(-)$ \\
\hline $250 \mu \mathrm{M}$ sulfamethoxazole $+1 \mathrm{mM}$ adenosine & $4(+++)$ & $4(+)$ \\
\hline $500 \mu \mathrm{M}$ sulfamethoxazole $+1 \mathrm{mM}$ adenosine & $2(++)$ & $2(+)$ \\
\hline $500 \mu \mathrm{M}$ sulfamethoxazole $+1 \mathrm{mM}$ adenosine $+5 \mu \mathrm{M}$ azaserine & ND & $<2(+)$ \\
\hline $500 \mu \mathrm{M}$ sulfamethoxazole $+1 \mathrm{mM}$ adenosine $+15 \mu \mathrm{M}$ azaserine & $<2(+)$ & ND \\
\hline
\end{tabular}

${ }^{*}$ The median values of cytotoxic titres $(\mathrm{CU} / 50 \mu \mathrm{l})$ from at least three independent experiments.

${ }^{\dagger}$ Bacterial growth is represented according to the maximum $\mathrm{OD}_{560}$ values:,$+++>0.5 ;++, 0.25-0.5 ;+,<0.25 ;-$, no growth or no toxin production was observed; ND, not determined. 


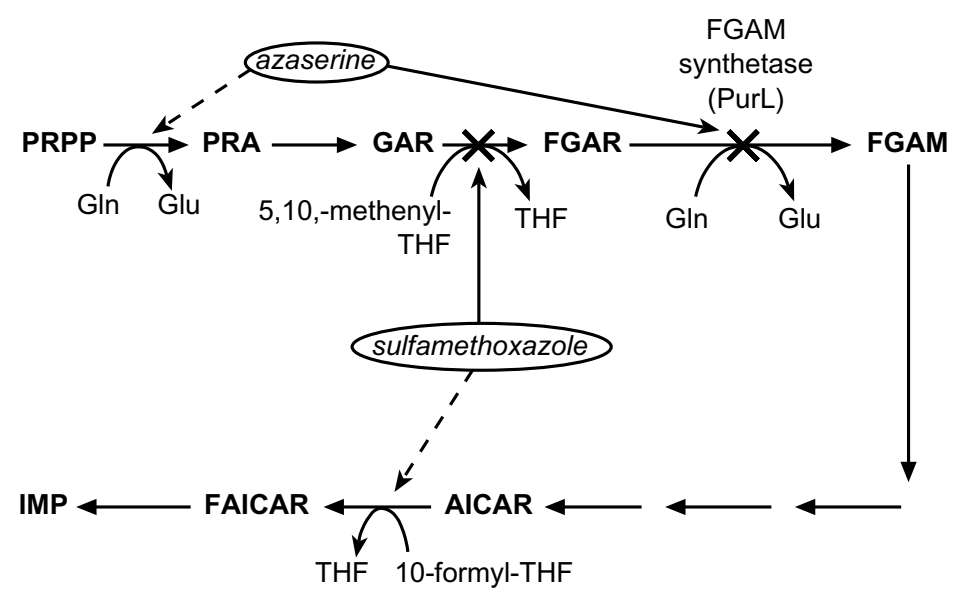

Fig. 6. Schematic representation of de novo purine biosynthesis. A combination of a cross and an arrow indicates the inhibition step by azaserine or sulfamethoxazole. Dotted arrows indicate the inconsistent inhibitions (see text). PRPP, phosphoribosylpyrophosphate; PRA, phosphoribosylamine; GAR, glycinamide ribonucleotide; FGAR, formylglycinamide ribonucleotide; FGAM, formylglycinamidine ribonucleotide; AICAR, aminoimidazolecarboxamide ribonucleotide; FAICAR, formamidoimidazolecarboxamide ribonucleotide; IMP, inosine monophosphate; THF, tetrahydrofolic acid.

catalyses the irreversible conversion of formylglycinamide ribonucleotide (FGAR), glutamine and ATP to FGAM, glutamate, ADP and orthophosphate. In $B$. subtilis and many other species of eubacteria and archaebacteria, FGAM synthetase is composed of two polypeptides: FGAM synthetase I (PurQ), which provides the glutamine amide transferase activity, and FGAM synthetase II (PurL), which provides the aminator activity [29]. On the other hand, in E. coli and a few other gram-negative eubacteria, this enzyme is encoded by a single gene, purL [30]. Interestingly, the FGAM synthetase of $C$. difficile described here consisted of a single polypeptide, the same as in E. coli (Fig. 2). To our knowledge, this is the first description of a gram-positive eubacterium that has a single geneencoded FGAM synthetase.

The expression pattern of the FGAM synthetase protein (PurL) was quite different between the biotin-sufficient and biotin-limited conditions (Figs. 1 and 4). Generally, all the purine biosynthesis activity would be coordinated with the growth phase and be stimulated in the exponential phase, because proliferating cells need to replicate their genomes and to produce many RNAs (mRNAs, rRNAs and tRNAs) to distribute them to newly generated cells. In biotin-sufficient conditions, the expression of PurL may be co-regulated with the entire activity of purine biosynthesis (Fig. 4a). However, in biotin-limited conditions, the expression of PurL did not appear to be in accordance with the growth phase. It was expressed continuously even after growth reached the stationary phase (Fig. 4b).

The inhibition of the enzymic activity of PurL by adding azaserine to the biotin-sufficient culture showed two characteristic responses, which were the same as the responses observed in the biotin-limited condition, i.e., enhanced toxin B production and PurL expression (Table 1 and Fig. 5). Azaserine is a diazo analogue of
L-glutamine. It competes with glutamine, which is a co-substrate of FGAM synthetase, and causes irreversible inhibition of the enzyme [28,31]. Azaserine inhibits many glutamine-dependent amidotransferases in vitro, but the most sensitive target is FGAM synthetase [31,32]. Furthermore, in vivo, azaserine inhibits mainly FGAM synthetase [28], therefore, it can be used as a specific inhibitor of FGAM synthetase. The essential effect of azaserine can be regarded as the resulting unavailability of glutamine. Likewise, the limitation of biotin can be considered to cause a shortage of glutamine because the biotin effect was neutralised by adding glutamine (Table 1) [14] and the expression of PurL was down-regulated by the addition of glutamine to the biotin-limited culture (Fig. 5, lane 5). Therefore, we concluded that the azaserine model was fundamentally equivalent to the biotin effect: the enhancement of toxin production under biotin-limited conditions was caused by inhibition of the enzymic activity of PurL, expression of which was up-regulated to recover the entire enzymic activity.

C. difficile cells in biotin-limited culture tend to lyse more rapidly after reaching maximum growth than cells in the biotin-sufficient culture (data not shown). There will be significant amounts of nucleic acidrelated compounds in such biotin-limited culture supernates. This culture state seems to correspond to that of the biotin-sufficient culture supplied with azaserine and hypoxanthine, or adenosine, in which toxin B production was enhanced more potently than it was in the biotin-sufficient culture supplied with azaserine only (Table 1). In other words, the biotinsufficient condition supplied with azaserine and hypoxanthine, or adenosine, mimics the biotin-limited condition more exactly with respect to toxin production. However, an important cause of the enhancement of toxin production is the inhibition of PurL due to the shortage of glutamine, because adding purine com- 
pounds alone did not have significant effects on toxin production. This extra enhancement occurred only when the PurL was inhibited.

The blockade of an enzyme results in the accumulation of metabolites prior to the blockade and depletion of subsequent metabolites. Blocking FGAM synthetase by azaserine causes the accumulation of FGAR [28, 31]. If the accumulation of FGAR or the depletion of the metabolites downstream of PurL participates in toxin production, blocking another step in the pathway could cause changes in toxin production. The addition of sulfamethoxazole at relatively high concentrations prevented toxin B production almost completely even in the biotin-limited or azaserine-added conditions (Table 1). In such concentrations, probably due to the almost complete inhibition of de novo purine synthesis, $C$. difficile cannot grow without a supply of adenosine. Sulfonamide inhibits folic acid biosynthesis and results in the inhibition of folic acid-dependent one-carbon transfer reactions. Therefore, sulfamethoxazole can inhibit the third and the ninth steps of the pathway that correspond to one step preceding and five steps following the step catalysed by FGAM synthetase, respectively (Fig. 6). However, the effect on the ninth step must be excluded, because this effect does not occur with the depletion of the metabolites downstream of PurL in the azaserine-added condition, even though toxin production was inhibited even in this condition (Table 1). Thus, the third step should be the main victim of sulfamethoxazole, and this blockade would cause the depletion of FGAR, in contrast to the blockade of PurL. In addition to the inhibitory effect on toxin production, the expression of PurL was reduced by adding sulfamethoxazole to the biotinlimited culture (Fig. 5, lane 4). These results suggest that the depletion of FGAR alone can neutralise the enhancement of toxin production and PurL expression even in biotin- and glutamine-deficient conditions. Therefore, the most plausible candidate for the signal that causes the enhancement of toxin production and PurL expression is the accumulation of FGAR rather than the shortage of glutamine or biotin.

In summary (Fig. 7), the shortage of glutamine caused by the limitation of biotin or the unavailability of glutamine caused by the addition of azaserine inhibits the enzymic activity of PurL. The resultant accumulation of FGAR enhances toxin production, and this enhancement is accelerated if exogenous purines are available. Simultaneously, the expression of PurL is upregulated in response to the accumulation of FGAR, but the entire enzymic activity is not recovered because of the shortage or unavailability of glutamine. Dissolution of the accumulation of FGAR neutralises both enhanced toxin production and PurL expression, which can be accomplished two ways: one is supplementation with glutamine, which causes the consumption of FGAR by restoration of the enzymic activity of PurL, and the other is addition of sulfamethoxazole, which

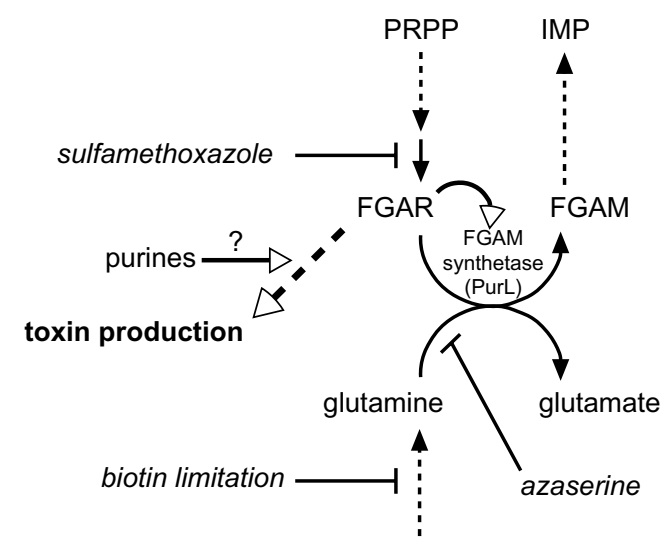

Fig. 7. A model for the effects of extrinsic factors on toxin production caused by altering the concentration of FGAR. The positive and negative influences are indicated by unfilled and flattened arrowheads, respectively. PRPP, phosphoribosylpyrophosphate; FGAR, formylglycinamide ribonucleotide; FGAM, formylglycinamidine ribonucleotide; IMP, inosine monophosphate.

prevents the synthesis of FGAR. It is interesting that toxin production by $C$. difficile is so tightly connected with purine metabolism via a metabolite, FGAR. The accumulation of FGAR carries at least two kinds of information, a shortage of glutamine or a disorder of purine synthesis, either or both of which would be disadvantageous to the vegetative growth of $C$. difficile. Therefore, it seems reasonable to use the accumulation of FGAR as a signal for toxin production. Attacking host cells by toxins would make it possible for $C$. difficile to obtain the required nutrients.

Experiments aimed at the disruption or overexpression, or both, of purL in C. difficile would help to confirm this hypothesis. However, there are few available methods for genetic manipulation of $C$. difficile, so appropriate vector systems are required to overcome this limitation. Attempts at the direct measurement of FGAR and the analysis of the process by which FGAR stimulates toxin production are underway in this laboratory.

In this study, the assay of toxin A was omitted in some experiments because the production of toxin A by ordinary toxigenic $C$. difficile strains has always paralleled that of toxin $B$ in this laboratory [13, $14,33]$. Other investigators found that the genes of toxins A and B were transcribed simultaneously [34, 35]. Therefore, this parallelism could be considered a general trait among strains that produce both toxins.

The results in this study also contain an important suggestion about the possibility of using sulfonamides as therapeutic agents for CDAD or PMC. Although $C$. difficile strains are highly resistant to sulfamethoxazole [19], the possibility that the treatment of CDAD or PMC could be accomplished by inhibiting toxin production by sulfonamides should be considered. In such a case, there would be few selective pressures, so 
that strains able to produce the toxins even in a sulfonamide-containing environment would not develop easily.

This work was supported in part by a Grant-in-Aid for Scientific Research from the Ministry of Education, Culture, Sports, Science and Technology of Japan, and by the Waksman Foundation of Japan, Inc. We dedicate this work to the memory of Dr Kiyotaka Yamakawa.

\section{References}

1. Sullivan NM, Pellett S, Wilkins TD. Purification and characterization of toxins $\mathrm{A}$ and $\mathrm{B}$ of Clostridium difficile. Infect Immun 1982; 35: 1032-1040.

2. Banno Y, Kobayashi T, Kono H, Watanabe K, Ueno K, Nozawa Y. Biochemical characterization and biologic actions of two toxins (D-1 and D-2) from Clostridium difficile. Rev Infect Dis 1984; 6 Suppl 1: S11-S20.

3. Lyerly DM, Krivan HC, Wilkins TD. Clostridium difficile: its disease and toxins. Clin Microbiol Rev 1988; 1: 1-18.

4. Dove $\mathrm{CH}$, Wang S-Z, Price SB et al. Molecular characterization of the Clostridium difficile toxin A gene. Infect Immun 1990; 58: 480-488.

5. Barroso LA, Wang S-Z, Phelps CJ, Johnson JL, Wilkins TD. Nucleotide sequence of Clostridium difficile toxin B gene. Nucleic Acids Res 1990; 18: 4004.

6. von Eichel-Streiber C, Laufenberg-Feldmann R, Sartingen S, Schulze J, Sauerborn M. Comparative sequence analysis of the Clostridium difficile toxins A and B. Mol Gen Genet 1992; 233: $260-268$.

7. Just I, Selzer J, Wilm M, von Eichel-Streiber C, Mann M, Aktories K. Glucosylation of Rho proteins by Clostridium difficile toxin B. Nature 1995; 375: 500-503.

8. Just I, Wilm M, Selzer J et al. The enterotoxin from Clostridium difficile (ToxA) monoglucosylates the Rho proteins. J Biol Chem 1995; 270: 13932-13936.

9. von Eichel-Streiber C, Boquet P, Sauerborn M, Thelestam M. Large clostridial cytotoxins - a family of glycosyltransferases modifying small GTP-binding proteins. Trends Microbiol 1996; 4: $375-382$.

10. Onderdonk AB, Lowe BR, Bartlett JG. Effect of environmental stress on Clostridium difficile toxin levels during continuous cultivation. Appl Environ Microbiol 1979; 38: 637-641.

11. Nakamura S, Mikawa M, Tanabe N, Yamakawa K, Nishida S. Effect of clindamycin on cytotoxin production by Clostridium difficile. Microbiol Immunol 1982; 26: 985-992.

12. Honda T, Hernadez I, Katoh T, Miwatani T. Stimulation of enterotoxin production of Clostridium difficile by antibiotics. Lancet 1983; 1: 655.

13. Yamakawa K, Karasawa T, Ikoma S, Nakamura S. Enhancement of Clostridium difficile toxin production in biotin-limited conditions. J Med Microbiol 1996; 44: 111-114.

14. Yamakawa K, Karasawa T, Ohta T, Hayashi H, Nakamura S. Inhibition of enhanced toxin production by Clostridium difficile in biotin-limited conditions. J Med Microbiol 1998; 47: $767-771$.

15. Karasawa T, Maegawa T, Nojiri T, Yamakawa K, Nakamura S. Effect of arginine on toxin production by Clostridium difficile in defined medium. Microbiol Immunol 1997; 41: 581-585.

16. Ikeda D, Karasawa T, Yamakawa K, Tanaka R, Namiki M, Nakamura S. Effect of isoleucine on toxin production by Clostridium difficile in a defined medium. Zentralbl Bakteriol 1998; 287: 375-386.
17. Karlsson S, Burman LG, Akerlund T. Suppression of toxin production in Clostridium difficile VPI 10463 by amino acids. Microbiology 1999; 145: 1683-1693.

18. Nakamura S, Mikawa $\mathrm{M}$, Nakashio $\mathrm{S}$ et al. Isolation of Clostridium difficile from the feces and the antibody in sera of young and elderly adults. Microbiol Immunol 1981; 25: 345-351.

19. Nakamura S, Nakashio S, Mikawa M, Yamakawa K, Okumura S, Nishida S. Antimicrobial susceptibility of Clostridium difficile from different sources. Microbiol Immunol 1982; 26: 25-30.

20. Karasawa T, Ikoma S, Yamakawa K, Nakamura S. A defined growth medium for Clostridium difficile. Microbiology 1995; 141: $371-375$.

21. Bradford MM. A rapid and sensitive method for the quantitation of microgram quantities of protein utilizing the principle of protein-dye binding. Anal Biochem 1976; 72: $248-254$

22. Sambrook J, Fritsch EF, Maniatis T. Molecular cloning: a laboratory manual, 2nd edn. Cold Spring Harbor, NY, Cold Spring Harbor Laboratory Press. 1989.

23. Shyamala V, Ames GF. Genome walking by single-specificprimer polymerase chain reaction: SSP-PCR. Gene 1989; 84: $1-8$.

24. Shyamala V, Ames GF-L. Genome walking by single specific primer-polymerase chain reaction. Methods Enzymol 1993; 217: $436-446$

25. Hofmann K, Bucher P, Falquet L, Bairoch A. The PROSITE database, its status in 1999. Nucleic Acids Res 1999; 27: $215-219$.

26. Henikoff JG, Henikoff S, Pietrokovski S. New features of the Blocks Database servers. Nucleic Acids Res 1999; 27: 226-228.

27. Corpet F, Gouzy J, Kahn D. Recent improvements of the ProDom database of protein domain families. Nucleic Acids Res 1999; 27: 263-267.

28. Lyons SD, Sant ME, Christopherson RI. Cytotoxic mechanisms of glutamine antagonists in mouse L1210 leukemia. J Biol Chem 1990; 265: 11377-11381.

29. Ebbole DJ, Zalkin H. Cloning and characterization of a 12 gene cluster from Bacillus subtilis encoding nine enzymes for de novo purine nucleotide synthesis. J Biol Chem 1987; 262: 8274-8287.

30. Schendel FJ, Mueller E, Stubbe J, Shiau A, Smith JM. Formylglycinamide ribonucleotide synthetase from Escherichia coli: cloning, sequencing, overproduction, isolation, and characterization. Biochemistry 1989; 28: 2459-2471.

31. Levenberg B, Melnick I, Buchanan JM. Biosynthesis of the purines, XV. The effect of aza-L-serine and 6-diazo-5-oxo-Lnorleucine on inosinic acid biosynthesis de novo. $J$ Biol Chem 1957; 225: 163-176.

32. Livingston RB, Venditti JM, Cooney DA, Carter SK. Glutamine antagonists in chemotherapy. Adv Pharmacol Chemother 1970; 8: 57-120.

33. Yamakawa K, Kamiya S, Meng XQ, Karasawa T, Nakamura S. Toxin production by Clostridium difficile in a defined medium with limited amino acids [published erratum appears in $J$ Med Microbiol 1995; 43: 461]. J Med Microbiol 1994; 41: 319-323.

34. Hammond GA, Lyerly DM, Johnson JL. Transcriptional analysis of the toxigenic element of Clostridium difficile Microb Pathog 1997; 22: 143-154.

35. Hundsberger T, Braun V, Weidmann M, Leukel P, Sauerborn M, von Eichel-Streiber C. Transcription analysis of the genes tcdA-E of the pathogenicity locus of Clostridium difficile. Eur $J$ Biochem 1997; 244: 735-742. 